\title{
38 GHz Antennas on Micromachined Silicon Substrates.
}

\author{
Romolo Marcelli ${ }^{(1)}$, Mircea Dragoman ${ }^{(2)}$, Dan Neculoiu ${ }^{(3)}$, \\ Flavio Giacomozzi ${ }^{(4)}$, Alexandru Müller ${ }^{(2)}$, N. Nitescu ${ }^{(2)}$ \\ ${ }^{(1)} \mathrm{M}^{2} \mathrm{~T}$ - Microwave Microsystems Technology \\ CNR - Institute for Microelectronics and Microsystems, Rome Section \\ Via del Fosso del Cavaliere 100, 00133 Roma, Italy \\ Phone: +3906 4993.4536; E-mail: r.marcelli@psm.rm.cnr.it \\ ${ }^{(2)}$ National Institute for Microsystem Technologies \\ 32B Erou Iancu Nicolae Str., 72990 Bucharest, Romania \\ Phone: +40 1 490.82.12 ; E-mail: mircead@imt.ro \\ (3)"Politehnica" University of Bucharest, Faculty of Electronics and Telecommunications \\ 1-3 Iuliu Manu Bd., 77752 Bucharest, Romania \\ Phone: +40 12312515 ; E-mail: dneculoiu@ @erra.gef.pub.ro \\ ${ }^{(4)}$ Istituto Trentino di Cultura - Istituto di Ricerca Scientifica e Tecnologica \\ Via Sommarive 18, 38050 Povo (TN), Italy \\ Phone: +39 0461 314432; E-mail: giaco@itc.it
}

\begin{abstract}
A new configuration of a double folded double slot CPW feed micromachined antenna array was realized on a $1.5 \mu \mathrm{m}$ thin three-layer dielectric membrane fabricated on a silicon substrate. The antenna was designed for an operating frequency of $38 \mathrm{GHz}$, and the double folded configuration was used for minimizing the membrane extension.
\end{abstract}

\section{INTRODUCTION}

Planar antennas are key elements in microwave and millimeter wave systems for many applications, such as wireless LAN communications, radio astronomy, automotive radars, remote sensing. Among many possible realizations the planar geometry allows to have full integrability with other passive components like filters, lumped elements, combining structures, and in particular with active devices. Moreover, it is possible to put a large number of radiators on the same substrate, thus allowing an easy realization of a phased array configuration.

In recent years, the micromachining technology has been proposed for the fabrication of millimeter wave circuits on very thin dielectric substrates (membranes) [1]. This kind of realization offers many advantages: the most important ones are the reduction of losses due to the decrease of the value of the dielectric permittivity, and the reduction of the dispersion effects. For the antennas there is also another important advantage: the suppression of unwanted substrate modes, which otherwise could lead to additional losses [2,3].

In this respect, a new configuration of a double folded slot CPW micromachined antenna array realized on a $1.5 \mu \mathrm{m}$ thin three-layer dielectric membrane is modelled and characterized at $38 \mathrm{GHz}$. 


\section{THEORY AND SIMULATIONS}

The proposed double folded double slot CPW feed micromachined antenna array is presented in Fig. 1 [4]. The membrane is centered around the 4 radiators of the array (grey colour in the figure) in an area of $6 \times 5 \mathrm{~mm}^{2}$, and the metallization is Au electroplated up to a thickness of $2 \mu \mathrm{m}$.

The entire array was realized on a bulk high resistivity $\langle 100\rangle$ Si substrate having $525 \mu \mathrm{m}$ thickness. The area delimited by the membrane was then anisotropically etched in order to release the $1.5 \mu \mathrm{m}$ thick $\mathrm{SiO}_{2} / \mathrm{Si}_{3} \mathrm{~N}_{4} / \mathrm{SiO}_{2}$ membrane. Each slot has a total length of about $4 \mathrm{~mm}$, i.e. $\lambda / 2$ the free space wavelength at $38 \mathrm{GHz}$ ca. The slot has the following dimensions: $\mathrm{x}=1.3 \mathrm{~mm}$ straight horizontal arm, a folded arm of $1.1 \mathrm{~mm}$ and is coupled with the CPW feed line over a distance of $\mathrm{y}=1.1 \mathrm{~mm}$ (the second folded arm). The realized configurations are shown in Fig. 2. In Fig. 3 it is shown the array mounted on the carrier used for E-plane and $\mathrm{H}$-plane radiation pattern measurements.

The antenna configuration was simulated by means of the Microwave Office-EMSight electromagnetic planar simulator and its predicted response for the current distribution is displayed in Fig. 4. From the above picture it can be observed the strong coupling between the slot and the the CPW feed and the maximum current distribution situated at the end of the slot. The current is uniformly distributed along each straight arm of the slot.

The folding of the slots was proposed to minimize the dimensions of the membrane, to avoid a too wide area for the suspended configuration. Despite such a constraint introduced for improving the reliability of the technological process, the folded slots are predicted to strongly radiate.

The modelled E-plane radiation pattern has shown a beamwidth of $60^{\circ}$ and a secondary lobe level less than $10 \mathrm{~dB}$, as it is shown in Fig. 5.

\section{EXPERIMENTAL RESULTS}

The designed micromachined antennas were tested in the E-plane and in the H-plane by means of a purposely set-up bench controlled by a HP8510C Network Analyzer. Two horn antennas are mounted on aluminium vertical columns fixed to an aluminium horizontal bar. A wood made box internally covered by microwave absorbing material surrounds the measurement area. The horn antennas (WR28 FL UBR320, $20 \mathrm{~dB}$ gain, FLANN material) are connected to WR22 FL UG$383 / \mathrm{U}-2.4 \mathrm{~mm}$ female VSWR 1.30 max adaptors from waveguide to $2.4 \mathrm{~mm}$ connectors. A goniometer at the base of the Aluminium columns supporting the antennas is used for changing the angle in the plane.

Measurements were performed at $38 \mathrm{GHz}$, the designed working frequency for such an array, and it has been demonstrated a good agreement between theory and experiment, as shown in the radiation patterns of Fig. 6 and Fig. 7 as compared to the results plotted in Fig. 5.

\section{CONCLUSIONS}

A micromachined antenna array consisting into 2 folded CPW slots was realized on a three-layer dielectric membrane and measured by means of a measurement set-up specially designed for this purpose. The results are in agreement with the predicted parameters obtained by electromagnetic modeling.

The antenna array is an original configuration realized on a thin membrane which has typical dimensions used in MEMS technology, but optimized by means of a folded configuration. Moreover, the $180^{\circ}$ rotation of the second antenna of the array allows the correct phase feed and the reduction of the antenna space occupancy. The array has been realized using standard Si technology and could be a perfect candidate for the required unexpensive but high performance antennas which are necessary for wireless communications assigned to the $38 \mathrm{GHz}$ bandwidth. 


\section{ACKNOWLEDGMENT}

Work supported by the INCO-COPERNICUS Project 977131 "MEMSWAVE".

\section{REFERENCES}

[1] Héctor J. De Los Santos: "Introduction to Microelectromechanical (MEM) Microwave Systems"; Artech House, London (1999).

[2] C. T.-C. Nguyen, L. Katehi, and G. Rebeiz: "Micromachined Devices for Wireless Communications"; Proceedings of IEEE, Special Issue on Integrated Sensors, Micromachining and Microsystems, Vol.86, No.8, pp.1756-1768 (1998)

[3] S. Perrot, Ch. Person, C. Quendo, and M. Ney: "Low Cost Millimeterwave Aperture Coupled Antenna Array on Polymer Membrane Substrate"; Proccedings of International Microwave Symposium (2000).

[4] SS. Garcia and JJ. Laurin: "Study of a CPW inductively coupled slot antenna"; IEEE AP 47, pp. 58-64 (1999).

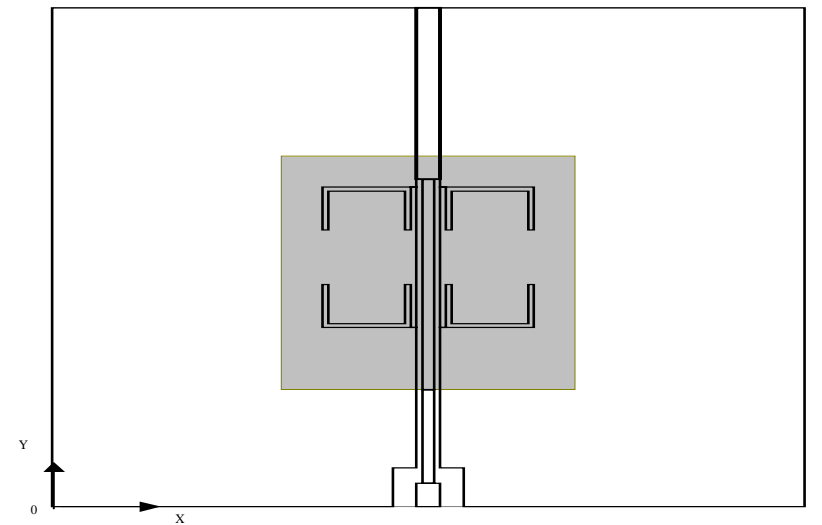

Fig.1. The array configuration and the membrane position on it.

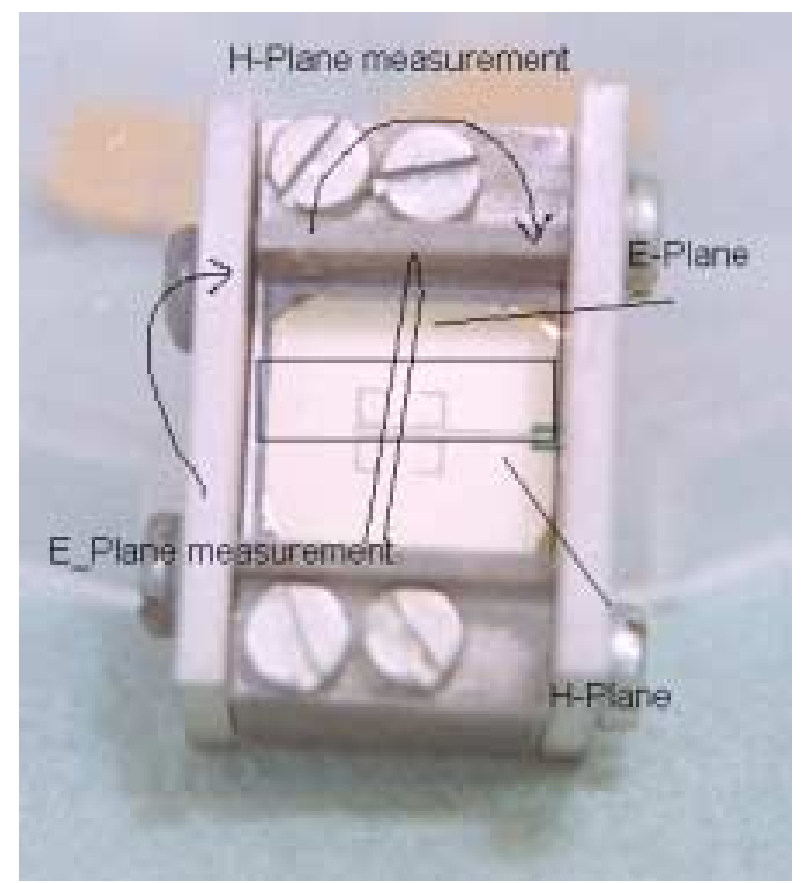

Fig.3. The assembled micromachined antenna, with E-plane and H-plane for the measurements.

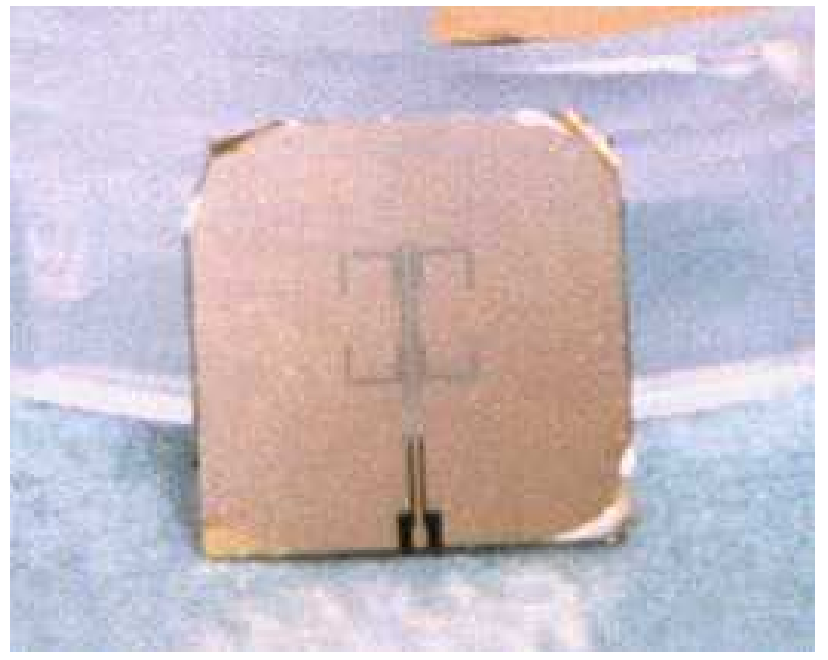

A)

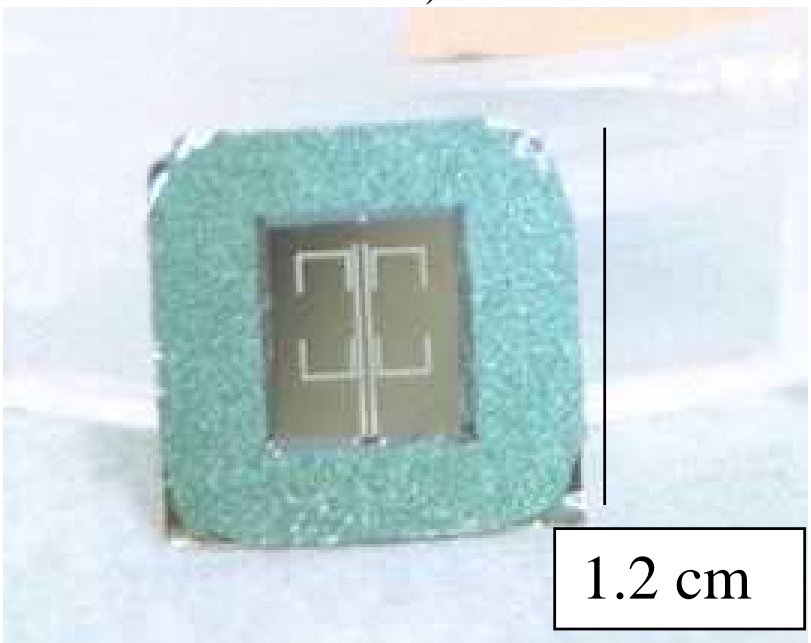

B)

Fig. 2. Practical realization of the micromachined antennas on a tri-layer dielectric membrane. In A) and B) the front and back photos of the single antenna are given respectively. 


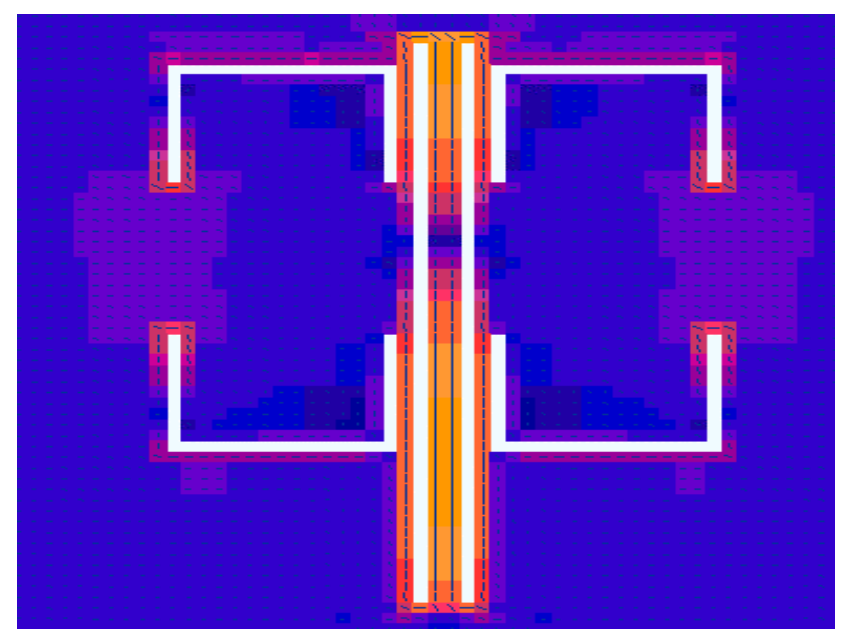

Fig. 4. The current distribution of the $38 \mathrm{GHz}$ array. A $4 \mathrm{~mm}$ high metal box was imposed for the boundary conditions, to be reproduced in the realization of the breadboard.
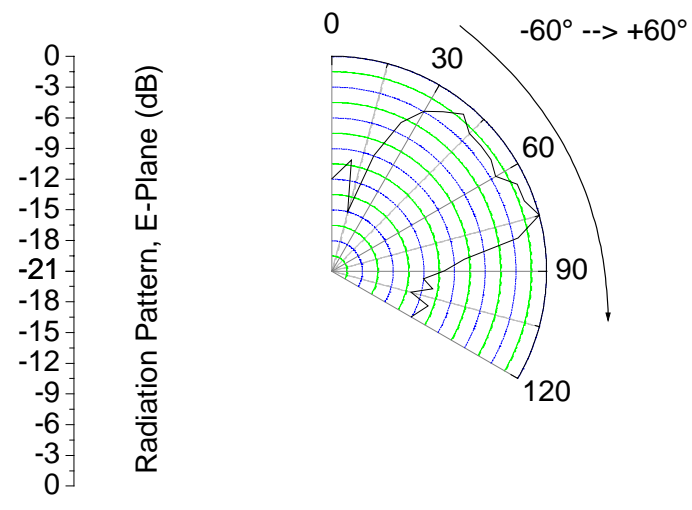

Fig. 6. Radiation pattern of the micromachined antenna measured at $38 \mathrm{GHz}$ in the E-plane.

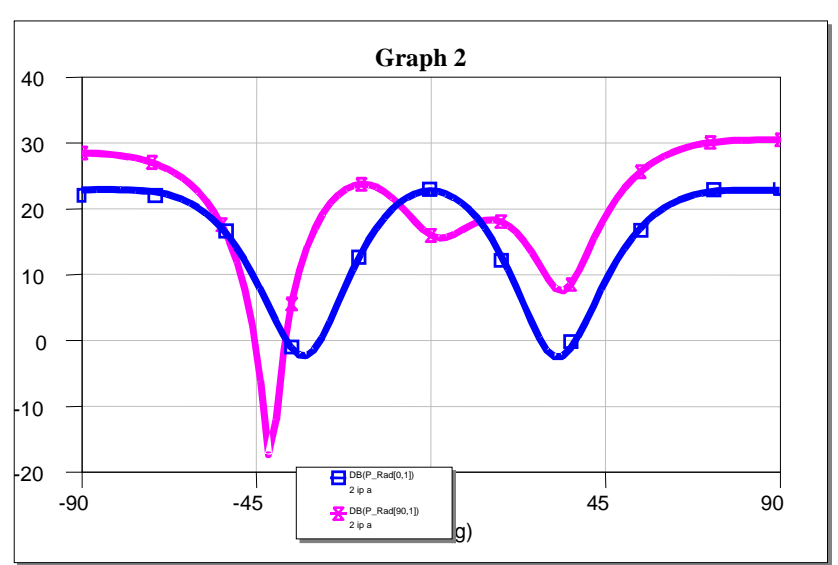

Fig. 5. Radiation pattern of the double-folded slot antenna array.
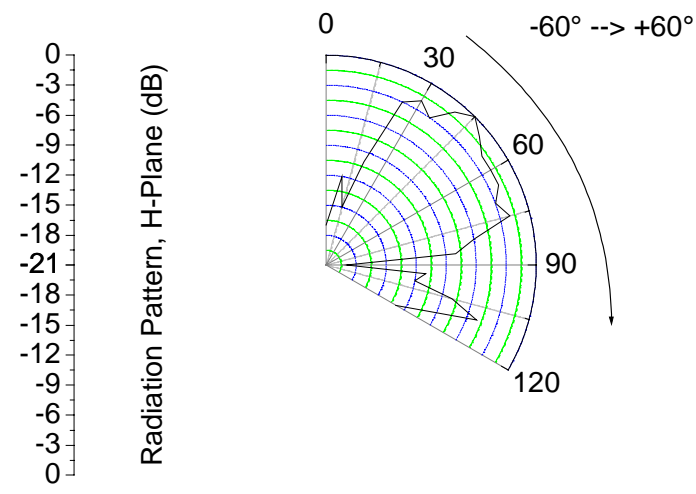

Fig. 7. Radiation pattern of the antenna measured in the H-plane. 